\title{
Proteomics of M-phase entry: 'Omen' vs. 'Omre', the battle for oocyte quality and beyond
}

\author{
Jacek Z. Kubiak \\ UMR 6061 CNRS/University Rennes 1, Institute of Genetics \& Development of Rennes (IGDR), \\ IFR 140 GFAS, Cell Cycle Group, France
}

\begin{abstract}
The understanding of cell cycle regulation benefited greatly from omic approaches. Because the cell cycle engine relies heavily on proteins, proteomic methods play a key role in identification of cell cycle players. The proteomic approach delivers an enormous volume of data, but it often lacks comprehensiveness. To ensure the comprehensiveness of results the discovery of novel proteins must be followed by functional analysis. Using Xenopus laevis oocytes in two different proteomic screens, we have recently identified a number of proteins whose behavior suggested specific and unexpected roles in M-phase entry. Functional analysis of EP45 identified in one of these screens has shown that M-phase entry is stimulated by Oocyte-Maturation-ENhancer ('Omen') activity. The second screen suggests the presence of an antagonistic activity, which we call 'Omre' (Oocyte-Maturation-REpressor). The equilibrium between Omen and Omre activities may determine the quality of oocytes and further embryo development via participation in making the decision whether to enter oocyte maturation. It remains an open question whether similar activities operate during mitotic divisions in embryonic and adult cells. Identifying such activities in somatic cells might impact on cancer treatments. (Folia Histochemica et Cytobiologica 2011, Vol. 49, No. 1, 1-7)
\end{abstract}

Key words: Bet-hedging, cell cycle, EP45, M-phase entry, oocyte, proteomics, Xenopus laevis

\section{Introduction}

The precise control of the M-phase in each cell cycle is of crucial importance for correct cell cycle progression. Cell division allows the separation of DNA duplicated during the S-phase of the cell cycle. Besides DNA, cytoplasmic organelles must also be correctly distributed between two daughter cells upon cell division. Centrioles are the crux of the microtubule-organizing centrosomes, and through this activity control the whole architecture of the cell, duplicating synchronously with nuclear DNA. Accordingly, they are distributed with great precision, and synchronously with chromosomes, to the daughter cells. Recent studies have shown that the link be-

Correspondence address: J.Z. Kubiak,

UMR 6061 CNRS/University Rennes 1, Institute of Genetics \& Development of Rennes (IGDR), Cell Cycle Group, France; tel.: (+ 33 02) 232346 98, fax: (+ 33 02) 232344 78;

e-mail: jacek.kubiak@univ-rennes1.fr tween centrosomal and DNA cycles may be even closer than previously thought [1]. Apparently, the segregation of other organelles such as Golgi apparatus, mitochondria, lysosomes or ER, seems to require less control (Figure 1). However, even their partition is strictly regulated and monitored during the M-phase. All these dynamic changes require temporal and spatial regulation, which is executed by appropriate regulatory proteins.

Thus, the key to understanding M-phase regulation lies in understanding regulatory proteins, their interactions and interrelations.

Mitotic regulatory pathways began to be elucidated when Yoshio Masui and Clement Markert discovered the existence of the major M-phase regulator, the M-phase Promoting Factor, or MPF [2]. Their seminal study was performed on Xenopus laevis oocytes and applied micromanipulations, including the transfer of cytoplasm from one oocyte to another. Their main conclusion was that the cytoplasmic activity, which they called Maturation Promoting Fac- 


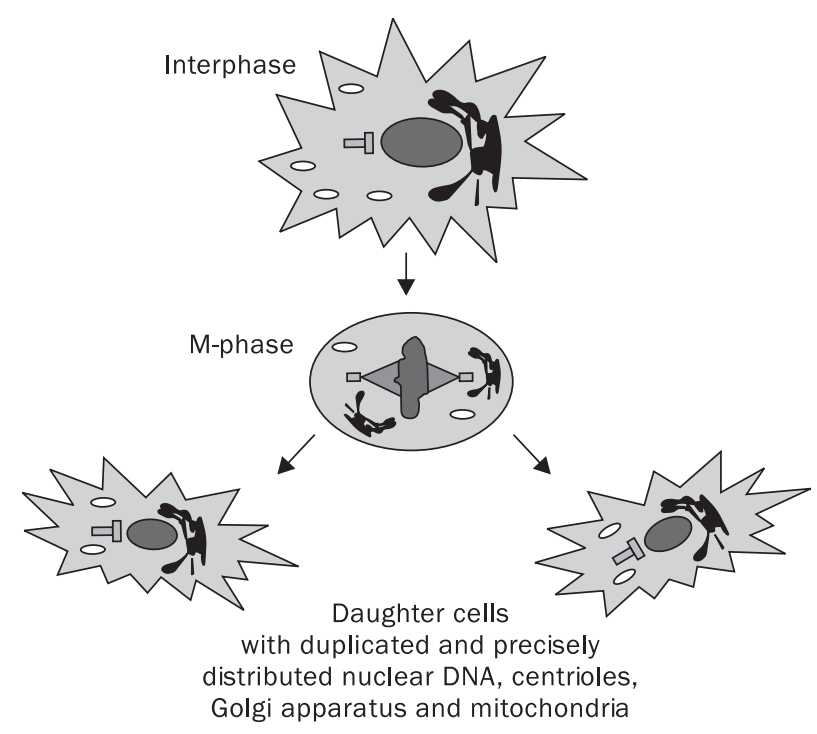

Figure 1. Interphase to M-phase transition and cell division

tor (MPF), induces M-phase entry. MPF was shown to be universal among all eukaryotic cells, from yeast to human. Because further studies showed that the same MPF induces not only meiotic but also mitotic M-phase, Maturation Promoting Factor became M-phase Promoting Factor.

MPF was further identified as a protein kinase, and more precisely a complex between cdc2/CDC28 gene product called CDK1 (Cyclin Dependent Kinase 1), which has an enzymatic activity, and the regulatory subunit of cyclin B $[3,4]$. This discovery was honored by the Nobel Prize in Physiology and Medicine for 2001 awarded to Paul Nurse and Tim Hunt. The third laureate of this prize, Leland Hartwell, discovered the enormous complexity of MPF activation and inactivation machinery controlled by cell cycle checkpoints [5].

Since those groundbreaking days, tools such as proteomics have been developed to study the players in the cell cycle. Proteomics allows the analysis of whole proteomes or selected sub-proteomes, so it is widely used to explore the protein composition of cells at different stages of the cell cycle, development or pathological states and/or upon various treatments. We used this approach to search for new mitotic regulators in Xenopus laevis oocytes.

\section{M-phase entry in oocytes: keys to activate MPF}

Xenopus oocyte maturation is an excellent model in which to study M-phase initiation by proteome approach due to the abundance of maternal proteins accumulated in the oocytes during oogenesis. Fully

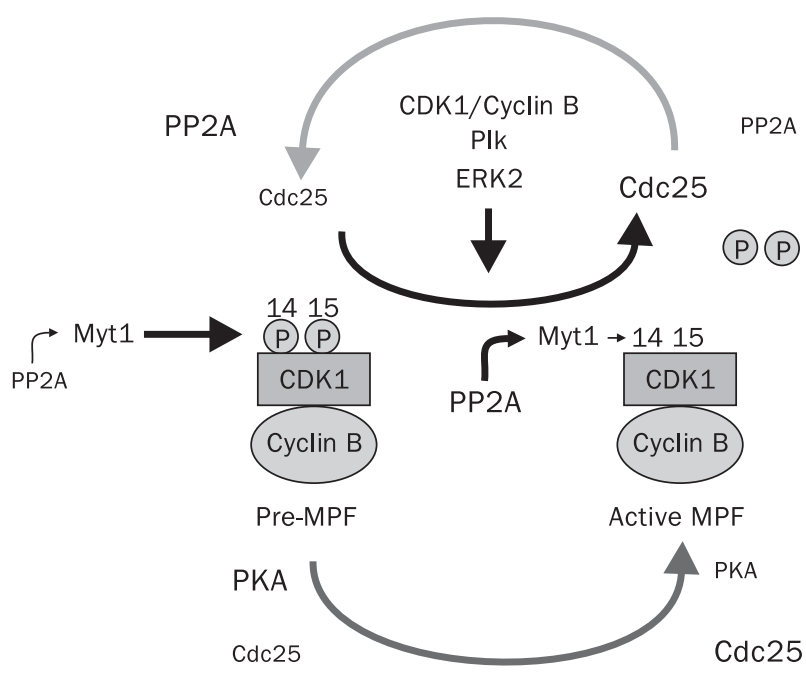

Figure 2. Major phosphorylation pathways involved in pre-MPF to MPF transition in Xenopus laevis oocytes

grown stage VI oocytes are arrested in meiotic prophase I, and wait for an external signal triggering initiation of the M-phase. In such immature oocytes, MPF is already present. However, it is maintained in an inactive form of pre-MPF because CDK1 in such a pre-MPF complex remains phosphorylated on its inhibitory sites of tyrosine 15 and threonine 14 by Myt1 kinase (Figure 2, left; [6]).

Activation of pre-MPF to active MPF occurs upon activation of a very specific phosphatase, Cdc25, dephosphorylating both tyrosine 15 and threonine 14 in concert with inhibition of Myt1 kinase [7]. Both Cdc25 and Myt1 are themselves regulated by phosphorylation. Two major kinases activating Cdc25 are CDK1 itself and polo-like kinase (Plk). The third kinase involved in the process is MAP kinase ERK2 (Extracellulary Regulated Kinase 2; [8]). As it usually happens in regulatory mechanisms implicating phosphorylation and dephosphorylation, the inhibition of Cdc25 and Myt1 relies on their dephosphorylation. This function is fulfilled by serine/threonine phosphatase PP2A [9]. Thus, the maintenance of the pre-MPF in its inactive state requires a well controlled equilibrium between phosphorylation and dephosphorylation of $\mathrm{Cdc} 25$ and Myt1 by the above-mentioned enzymes (Figure 2). The major upstream player in this process is cAMP-dependent protein kinase (PKA). As long as cAMP level remains high, PKA activity stays high too, and the oocyte is unable to activate MPF. A mixture of steroid hormones (among which progesterone seems to play a major role) delivered physiologically from follicular cells, or via experimental treatment in the case of oocytes isolated from the ovary, triggers a diminution of PKA activity [10-12]. 
The molecular mechanisms involved in the relationship between the inhibition of PKA and the activation of MPF is not fully understood (Figure 2). However, Duckworth et al. suggested that PKA participates in MPF down regulation during the prophase arrest of Xenopus laevis oocytes via phosphorylation of Serine 287 of Cdc25 which has an inhibitory effect on this phosphatase [13]. Thus, the modulation of PKA activity via a hormonally regulated mechanism seems key to the decision to maintain the prophase arrest or to activate MPF and to enter oocyte maturation.

A new protein synthesis is a parallel pathway to phosphorylation/dephosphorylation cascade. Protein synthesis inhibition (similarly to inhibition of phosphorylation) prevents MPF activation and oocyte maturation. One of the newly synthesized proteins central to MPF activation is a proto-oncogene called c-Mos. c-Mos is a serine/threonine kinase, which activates the MAP kinase pathway terminating by p90 $0^{\text {rsk }}$, another kinase phosphorylating Myt1 kinase. This phosphorylation is required for the inhibition of Myt1, in turn necessary for MPF activation [14]. Other key proteins whose synthesis is augmented by progesterone are the B-type cyclins. Their appearance is necessary to deliver more complexes with CDK1, which is in clear excess compared to cyclin B at the beginning of oocyte maturation.

The extremely rapid activation of MPF is ensured by a positive feedback between CDK1 and Cdc25. Even a small increase in the activity of CDK1/cyclin B complex, when single molecules of CDK1/cyclin B become active due to the appearance of new cyclin B molecules, induces Cdc25 and Plk phosphorylation. When this happens, the fragile equilibrium of the prophase arrest breaks down and the explosive activation of the remaining $\mathrm{CDK} 1 /$ cyclin $\mathrm{B}$ is triggered. This is the so-called auto-amplification loop of MPF (Figure 2, right; [15]).

Interestingly, functions of the neosynthesis of c-Mos and cyclin B are apparently redundant, and new synthesis of either of these proteins is sufficient to activate MPF and to begin oocyte maturation in Xenopus laevis [16]. Moreover, in the absence of newly synthesized c-Mos and cyclin B, the inhibition of PKA activity cannot trigger MPF activation [15]. This interrelation demonstrates that M-phase initiation in the Xenopus oocyte requires both the progesterone signal to inactivate PKA and the burst of c-Mos and cyclin B synthesis. This is despite the fact that in experimental conditions only one protein out of this pair (c-Mos or cyclin B) is absolutely necessary.

These results show the complexity of the machinery controlling M-phase entry in prophase oo- cytes. It is important to note that the pathways involved in Xenopus laevis oocyte maturation are both based on post-translational modifications of existing proteins (phosphorylation and dephosphorylation), as well as on the appearance of new actors (c-Mos and cyclin B).

This is not the case for all vertebrate species. For instance, in the mouse oocyte, a new protein synthesis is not necessary for activating MPF and triggering the meiotic M-phase. Apparently, a stock of cyclin B necessary to initiate the loop of MPF activation is sufficient to trigger the M-phase [17]. The new cyclin B synthesis is, however, necessary in mouse oocytes for further progression through the first meiotic M-phase [18].

Interestingly, younger Xenopus laevis oocytes, for instance in stage IV, although they morphologically resemble fully grown oocytes, and contain pre-MPF and Cdc25 phosphatase, are unable to respond to progesterone stimulation [19, 20]. More surprisingly, they possess progesterone receptors and even decrease the cAMP level upon progesterone action [21]. This shows that the cause of their inability to activate MPF is located downstream from the cAMP. Apparently, Plk is an enzyme lacking in these oocytes, which breaks the amplification loop of MPF, preventing c-Mos synthesis and down regulation of Myt $1[22,23]$. Since protein deficiency determines the state of oocyte responsiveness, a comparison between proteomes of stage IV and VI oocytes might well help with understanding the mechanisms involved in M-phase initiation.

\section{Comparison between proteomes of oocytes in stage IV and VI: Omen enters the stage}

Recently, we took advantage of this model system and compared proteomes of oocytes in stage IV and VI by a traditional 2D-gel electrophoresis, looking for proteins lacking in stage IV. We thought that the lack of some proteins in stage IV oocytes could be involved in the inability of these oocytes to enter the M-phase (Figure 3). Indeed, the 2D-analysis showed a couple of spots absent in the proteome of stage IV oocytes in contrast to stage VI.

We focused our attention on a family of four spots identified by mass spectrometry as EP45 (Estrogen-Regulated Protein 45) [24].

This protein was first identified in Xenopus laevis as an estrogen-induced protein secreted to the blood by the liver in co-ordination with vitellogenin, the main component of yolk in oocytes $[25,26]$. This characteristic suggested that EP45 could be uptaken by oocytes from the blood. Indeed, a recent proteomic 


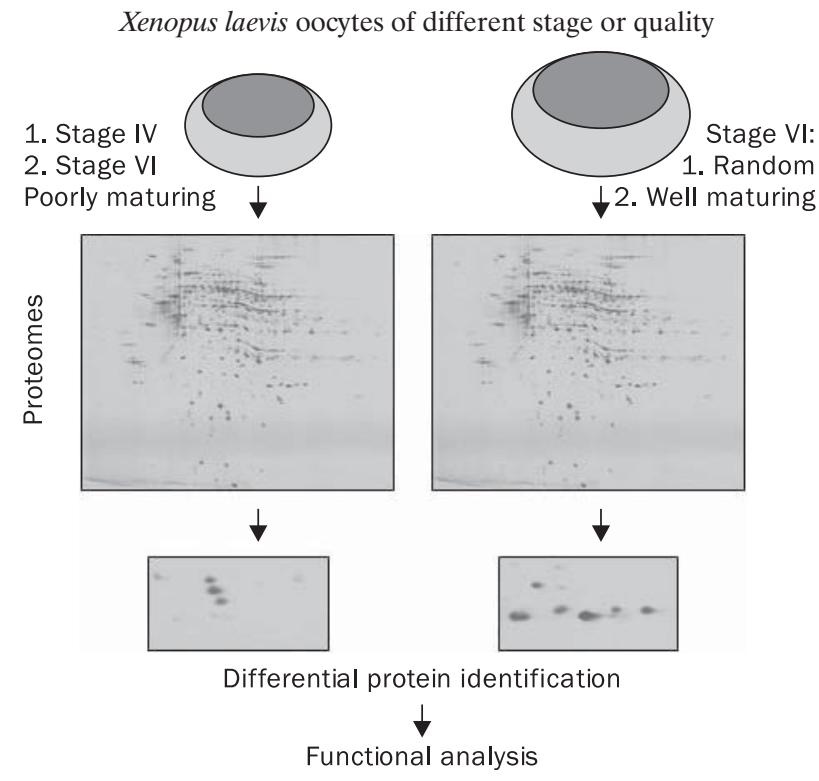

Figure 3. Differential proteomic screens comparing proteomes of (1): stage IV and VI and (2): stage VI poorly-maturing and well-maturing Xenopus leavis oocytes

study of yolk components in Xenopus laevis has shown that EP45 (under the new name of Seryp) is one of the major components of yolk granules and the major player in regulation of the source of energy necessary for embryo development [27]. On the basis of its amino acid sequence, EP45 was classified as a member of the serine protease inhibitors called Serpins $[26,27]$. EP45 has a histidine-rich N-terminus which determines its high affinity for nickel $[28,29]$. Thus, it was identified as a nickel-binding protein during studies focused on the teratogenic action of divalent nickel independently from early Holland's studies and named pNiXa (protein-Ni-Xenopus-a) [28]. pNiXa, purified from MII-arrested Xenopus laevis oocytes, was reported to induce oocyte maturation after injection into stage VI oocytes and its stimulatory action exhibited a synergistic effect with Ras-induced maturation [30].

This characteristic of $\mathrm{pNiXa}$ in conjunction with our proteomic screen showing that this protein accumulates in oocytes between stages IV and VI raised several questions. The first and most obvious was why an M-phase-inducing protein already present in oocytes does not provoke M-phase entry by itself?

Jorgensen et al. [27] and ourselves [24] have shown that EP45 is not synthesized in oocytes but in the liver and that it is delivered to the oocytes via blood and, probably, via follicular cells. We also have shown that over-expression of EP45 does not induce meiotic maturation either in stage IV or VI oocytes. The capacity previously reported by Haspel et al. [30] of pNiXa to induce M-phase entry in oocytes could occur due to contamination of the purified protein obtained from oocytes in MII, which have high activity of CDK1/cyclin B, and the whole machinery of the M-phase stabilization necessary during MII-arrest.

Most interestingly, the EP45 over-expression in oocytes responding moderately to progesterone clearly enhances M-phase entry [24]. This kind of M-phase-enhancing activity was previously unknown. We have named this novel activity of EP45 the 'oocyte maturation enhancement' activity or 'Omen'.

\section{Comparison between proteomes of well- -maturing and poorly-maturing oocytes: 'Omre' joins 'Omen'}

In parallel to the proteomic analysis of stage IV and VI oocytes, we focused on well-maturing and poorly-maturing stage VI oocytes [31]. We wanted to identify the proteins differentially expressed in oocytes of different quality. During oocyte growth, numerous proteins accumulate as a maternal store for future use by the developing embryo. The pattern of proteins accumulated in oocytes has been studied in different species, but no comparative studies between oocytes showing different capacities to enter into maturation, and thus different quality, has ever been carried out. As we have recently found the Omen activity enhancing M-phase entry and oocyte maturation, we could expect the presence of an opposite activity - Omre for "Oocyte maturation repressor”. Thus we were interested in identification of proteins specifically present or abundant in poorly-maturing oocytes, because such proteins could have an activity anatgonistic to the Omen activity. In our differential screen we found two spots specifically present in poorly-maturing oocytes, which were identified as two proteins: Elongation Factor 2 (EF2) and S-adenosyl-L-homosysteine hydrolase (SAHH).

These two spots, present specifically in 2-D gels made of poorly-maturing oocytes, most probably represent specific forms of the two proteins. It is difficult to imagine that a factor highly important to protein synthesis is absent from well-maturing oocytes. EF2 is known to be down regulated in stage VI Xenopus leavis oocytes via changes in its phosphorylation [32]. Thus, the spot found only in poorly-maturing oocytes suggests that EF2 may be modified in a specific way in these oocytes as opposed to well-maturing ones. SAHH is known as a highly conserved and ubiquitous enzyme hydrolyzing S-adenosylhomocysteine (SAH). SAH is a by-product of S-adenosylmethionine-dependent methylations [33]. The catalysis 
of this reaction makes SAHH a major positive regulator of methylation of DNA, RNA, lipids and proteins via decreasing the level of SAH [34]. Interestingly, SAHH and EF2 have been identified as proteins increased in another proteomic screen that looked at the response of rat astrocytes to oxidative stress induced by $\mathrm{H}_{2} \mathrm{O}_{2}$ [35]. Thus, the presence of the additional spots of each of these proteins in poorly-maturing oocytes could correlate with a response to a stress exercised on these oocytes. Similarly to EF2, SAHH is regulated by phosphorylation [36], which may result in its modified electrophoretic mobility in our 2-D gels. Thus, again only well-maturing oocytes may contain a specific isoform of SAHH correlating with the poor quality of oocytes.

\section{Energy and evolution: a battle between Omen and Omre}

Omre's potential activity needs to be verified by further functional analysis of selected proteins [31]. It is very tempting to imagine a battle between Omen and Omre activities which remain in equilibrium in physiologically normal cells (Figure 4).

The question also remains of how Omen activity interacts with CDK1-activating machinery. The fact that EP45 over-expression does not induce MPF activation in stage VI oocytes, and does not even accelerate oocyte maturation, suggests that this protein is not required for induction of the M-phase in physiological conditions. The clear enhancement of maturation upon suboptimal conditions, i.e. in oocytes in which a low progesterone dose cannot efficiently induce MPF activation, suggests that EP45 may act through an unspecific pathway downstream from progesterone receptors.

As EP45 has been shown to be involved in the regulation of yolk consumption in the embryo [27], we propose that it may ameliorate the efficiency of the

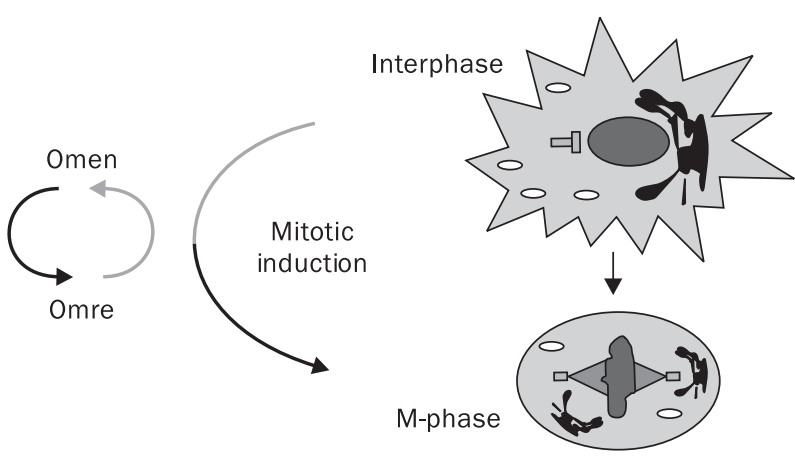

Figure 4. Hypothesis of the Omen versus Omre battle regulating interphase to $\mathrm{M}$-phase transition use of energy sources needed for the cell cycle transitions. Both nuclear envelope breakdown and chromatin assembly require adapted energy sources [37, 38]. It is also known that the meiotic program in yeast is under the control of sources of nutrients for energy production [39]. Moreover, it has been found in human oocytes that the number of mitochondria per oocyte, and thus probably oocyte efficiency to produce the energy, is linked to the quality of oocyte maturation: women suffering from ovarian insufficiency have low mtDNA content in oocytes [40]. Thus, EP45 over-expression may facilitate M-phase induction simply by restoring the energetic status quo in deficient or stressed oocytes. The fact that the action of EP45 is detectable only in poorly-maturing oocytes indicates that EP45-related activities ameliorate oocyte quality. This is an important observation, which shows that low quality oocytes have the ability to improve through the action of specific activities/proteins.

EP45 is certainly not the only protein involved in Omen activity. A number of similar activities and proteins may participate establishing the status of oocyte quality. Moreover, Omen activity may have equivalents in somatic cells, and as such may influence the ability and/or the timing of cells to enter the M-phase. Knowledge of such molecules may impact cancerology since the regulation of anarchic cell divisions in tumor cells is one of the pharmacological goals in the fight against cancer. Interestingly, the group of William Sunderman has shown that $\mathrm{pNiXa}$ antibody gave a positive staining in 21 of 181 human neoplasms, including prostate, liver, colon, and breast tumors, whereas the signal was absent in 119 specimens of normal human tissues [41]. Thus an EP45-related serpin (there is no clear EP45 homolog among human serpins) may be associated with certain tumorigenesis. This may happen via its Omen activity. The possibility of influencing the ability of tumor cells to divide via manipulation of Omen and Omre activities may open a new avenue in the battle against cancer.

Finally, the discovery of EP45 Omen activity could impact our view of the mechanisms of evolution. Under changing environmental conditions (e.g. following the abrupt appearance of hormonal disruptors in water), the level of progesterone in Xenopus laevis females may fall drastically. Such a change in hormonal regulation may provoke a fall in the number of oocytes undergoing normal meiotic maturation. This in turn would reduce the reproductive success of females. In such a case, the originally neutralfor-reproduction EP45-dependent processes become important survival factors, since they may ameliorate the quality of oocytes, embryo development and reproductive success. 
Such a mechanism resembles an evolutionary strategy well described in bacteria called bet-hedging, which is based on the stochastic presence of factors increasing survival in a drastically changing environment [42].

To the best of our knowledge, EP45 is the first gene product in vertebrates which could be directly involved in a bet-hedging strategy. Omen and Omre activities are very good candidates for factors essential for a bet-hedging strategy, because of their neutral character in normal physiological conditions. The potential battle between Omen and vs. Omre could thus be of significant importance for evolution.

In conclusion, a proteomic approach may help discover not only new proteins, but also new functions and activities of those proteins. The presence of 'hidden' activities such as Omen and Omre in oocytes shows the necessity of developing methods to identify and quantify proteins in individual oocytes. This could enable a comparison of the proteomes of selected cells in the search for proteins involved in the quality of oocytes. We are currently working on the development of approaches enabling the analysis of proteomes in single cells.

\section{Acknowledgements}

The author acknowledges the support provided by grants from Association pour la Recherche contre le Cancer (ARC) and Ligue Contre le Cancer (LCC) during writing this paper.

\section{References}

1. Ferguson RL, Maller JL. Centrosomal localization of cyclin E-Cdk2 is required for initiation of DNA synthesis. Curr Biol. 2010;20:856-860.

2. Masui Y, Markert CL. Cytoplasmic control of nuclear behavior during meiotic maturation of frog oocytes. J Exp Zool. 2010;177:129-145.

3. Evans T, Rosenthal ET, Youngblom J, Distel D, Hunt T. Cyclin: a protein specified by maternal mRNA in sea urchin eggs that is destroyed at each cleavage division. Cell. 1983;33:389-396.

4. Gautier J, Norbury C, Lohka M, Nurse P, Maller J. Purified maturation-promoting factor contains the product of a Xenopus homolog of the fission yeast cell cycle control gene cdc2+. Cell. 1988;54:433-439.

5. Hartwell LH, Weinert TA. Checkpoints: controls that ensure the order of cell cycle events. Science. 1989;246:629-634.

6. Dunphy WG, Newport JW. Fission yeast p13 blocks mitotic activation and tyrosine dephosphorylation of the Xenopus cdc2 protein kinase. Cell. 1989;58:181-191.

7. Gautier J, Solomon MJ, Booher RN, Bazan JF, Kirschner MW. cdc25 is a specific tyrosine phosphatase that directly activates p34cdc2. Cell. 1991;67:197-211.

8. Wang R, He G, Nelman-Gonzalez M, et al. Regulation of Cdc25C by ERK-MAP kinases during the G2/M transition. Cell. 2007;128:1119-1132.
9. Karaiskou A, Jessus C, Brassac T, Ozon R. Phosphatase 2A and polo kinase, two antagonistic regulators of cdc25 activation and MPF auto-amplification. J Cell Sci. 1999;112: 3747-3756.

10. Maller JL, Butcher FR, Krebs EG. Early effect of progesterone on levels of cyclic adenosine 3':5'-monophosphate in Xenopus oocytes. J BiolChem. 1979;254:579-582.

11. Maller JL, Krebs EG. Regulation of oocyte maturation. Curr Top Cell Regul. 1980;16:271-311.

12. Wang J, Liu XJ. Progesterone inhibits protein kinase A (PKA) in Xenopus oocytes: demonstration of endogenous PKA activities using an expressed substrate. J Cell Sci. 2004;117: 5107-5116.

13. Duckworth BC, Weaver JS, Ruderman JV. G2 arrest in Xenopus oocytes depends on phosphorylation of cdc25 by protein kinase A. Proc Natl Acad Sci USA. 2002;99:16794-16799.

14. Castro A, Peter M, Lorca T, Mandart E. c-Mos and cyclin B/ /cdc2 connections during Xenopus oocyte maturation. Biol Cell. 2001;93:15-25.

15. Jessus C, Ozon R. How does Xenopus oocyte acquire its competence to undergo meiotic maturation? Biol Cell. 2004; 96:187-192.

16. Haccard O, Jessus C. Redundant pathways for Cdc2 activation in Xenopus oocyte: either cyclin B or Mos synthesis. EMBO Rep. 2006;7:321-325.

17. Hoffmann S, Tsurumi C, Kubiak JZ, Polanski Z. Germinal vesicle material drives meiotic cell cycle of mouse oocyte through the 3»UTR-dependent control of cyclin B1 synthesis. Dev Biol. 2006;292:46-54.

18. Polanski Z, Ledan E, Brunet $\mathrm{S}$ et al. Cyclin synthesis controls the progression of meiotic maturation in mouse oocytes. Development. 1998;125:4989-4997.

19. Rime H, Yang J, Jessus C, Ozon R. MPF is activated in growing immature Xenopus oocytes in the absence of detectable tyrosine dephosphorylation of P34cdc2. Exp Cell Res. 1991;196:241-245.

20. Rime H, Jessus C, Ozon R. Tyrosine phosphorylation of p34cdc2 is regulated by protein phosphatase $2 \mathrm{~A}$ in growing immature Xenopus oocytes. Exp Cell Res. 1995;219: 29-38.

21. Mulner O, Belle R, Ozon R. cAMP-dependent protein kinase regulates in ovo cAMP level of the Xenopus oocyte: evidence for an intracellular feedback mechanism. Mol Cell Endocrinol. 1983;31:151-160.

22. Nakajima H, Toyoshima-Morimoto F, Taniguchi E, Nishida E. Identification of a consensus motif for Plk (Polo-like kinase) phosphorylation reveals Myt1 as a Plk1 substrate. Journal Biol Chem. 2003;278:25277-25280.

23. Karaiskou A, Lepretre AC, Pahlavan G, Du Pasquier D, Ozon $\mathrm{R}$, Jessus C. Polo-like kinase confers MPF autoamplification competence to growing Xenopus oocytes. Development. 2004;131:1543-1552.

24. Marteil G, D'Inca R, Pascal A et al. EP45 accumulates in growing Xenopus laevis oocytes and has oocyte-maturationenhancing activity involved in oocyte quality. J Cell Sci. 2010;123:1805-1813.

25. Holland LJ, Wangh LJ. Estrogen induction of a $45 \mathrm{kDa}$ secreted protein coordinately with vitellogenin in Xenopus liver. Mol Cell Endocrinol. 1987;49:63-73.

26. Holland LJ, Suksang C, Wall AA, Roberts LR, Moser DR, Bhattacharya A. A major estrogen-regulated protein secreted from the liver of Xenopus laevis is a member of the serpin superfamily. Nucleotide sequence of cDNA and hormonal induction of mRNA. J Biol Chem. 1992;267: 7053-7059. 
27. Jorgensen P, Steen JA, Steen H, Kirschner MW. The mechanism and pattern of yolk consumption provide insight into embryonic nutrition in Xenopus. Development. 2009; 136:1539-1548.

28. Beck BL, Henjum DC, Antonijczuk Ket al. pNiXa, a Ni(2+)-binding protein in Xenopus oocytes and embryos, shows identity to Ep45, an estrogen-regulated hepatic serpin. Res Commun Chem Pathol Pharmacol. 1992;77:3-16.

29. Sunderman FW Jr, Varghese AH, Kroftova OS et al. Characterization of pNiXa, a serpin of Xenopus laevis oocytes and embryos, and its histidine-rich, Ni(II)-binding domain. Mol Reprod Dev. 1996;44:507-524.

30. Haspel J, Sunderman FW Jr, Hofper SM et al. A nickel-binding serpin, $\mathrm{pNiXa}$, induces maturation of Xenopus oocytes and shows synergism with oncogenic ras-p21 protein. Res Commun Chem Pathol Pharmacol. 1993;79:131-140.

31. D'Inca R, Marteil G, Bazile F et al. Proteomic screen for potential regulators of M-phase entry and quality of meiotic resumption in Xenopus laevis oocytes. J Proteomics. 2010;73:1542-1550.

32. Severinov KV, Melnikova EG, Ryazanov AG. Downregulation of the translation elongation factor 2 kinase in Xenopus laevis oocytes at the final stages of oogenesis. New Biol. 1990;2:887-893.

33. Ueland PM. Pharmacological and biochemical aspects of S-adenosylhomocysteine and S-adenosylhomocysteine hydrolase. Pharmacol Rev. 1982; 34: 223-253.
34. Radomski N, Kaufmann C, Dreyer C. Nuclear accumulation of S-adenosylhomocysteine hydrolase in transcriptionally active cells during development of Xenopus laevis. Mol Biol Cell. 1999;10:4283-4298.

35. Miura Y, Kano M, Abe K, Urano S, Suzuki S, Toda T. Agedependent variations of cell response to oxidative stress: proteomic approach to protein expression and phosphorylation. Electrophoresis. 2005;26:2786-2796.

36. Dreyer C. Differential accumulation of oocyte nuclear proteins by embryonic nuclei of Xenopus. Development. 1987;101:829-846.

37. Kmiec EB, Sekiguchi JM, Cole AD. Studies on the ATP requirements of in vitro chromatin assembly. Biochem Cell Biol. 1989;67:443-454.

38. Higa MM, Ullman KS, Prunuske AJ. Studying nuclear disassembly in vitro using Xenopus egg extract. Methods. 2006;39:284-290.

39. Jambhekar A, Amon A. Control of meiosis by respiration. Curr Biol. 2008;18:969-975.

40. May-Panloup P, Chrétien MF, Jacques C, Vasseur C, Malthičry Y, Reynier P. Low oocyte mitochondrial DNA content in ovarian insufficiency. Hum Reprod. 2005;20:593-597.

41. Yamase HT, Forouhar FA, Sojda DF et al. Immunoperoxidase staining of tumors by an antibody to Xenopus pNiXa. Ann Clin Lab Sci. 1996;26:243-251.

42. Beaumont HJ, Gallie J, Kost C, Ferguson GC, Rainey PB. Experimental evolution of bet hedging. Nature. 2009;462:90-93.

Submitted: 14 October, 2010

Accepted after reviews: 5 December, 2010 